\title{
Desempenho de pós larvas de cascudo preto (Rhinelepis aspera), alimentadas com naúplios de artemia e ração oferecida em saches
}

\section{Performance of post larvae of cascudo preto (Rhinelepis aspera), fed with artemia nauplii and ration offered in sachets}

\author{
Luis Ricardo Jayme Guerreiro ${ }^{1 *}$; Jeferson Antonio Dias Dias²; Darci Carlos \\ Fornari $^{3}$; Ricardo Pereira Ribeiro ${ }^{4}$; Marco Antonio Zanoni ${ }^{5}$
}

\begin{abstract}
Resumo
O objetivo deste trabalho foi avaliar o desempenho de pós larvas de $R$. aspera submetidas a dois tipos de dietas. O delineamento experimental foi inteiramente casualizado com dois tratamentos e oito repetições. A densidade de estocagem foi de sete pós larvas/litro. As dietas eram compostas por: A, ração em pó com $55 \%$ PB oferecida em saches de organza e B, 850 naúplios de artemia/pós larva, três vezes ao dia. Não houve diferenças significativas na sobrevivência $(P \geq 0,05)$ entre os tratamentos. A análise dos resultados das biometrias revelou existir diferenças significativas $(P \geq 0,05)$ entre as duas dietas, sendo que a ração fornecida em saches proporcionou as maiores médias de comprimento $(\mathrm{mm})$ e peso $(\mathrm{mg})$. $\mathrm{O}$ fator de condição alcançou valores superiores $(\mathrm{P} \geq 0,05)$ com a "dieta viva". Os resultados demonstraram que a utilização de saches preenchido com ração podem ser uma boa alternativa para a larvicultura do Rhinelepis aspera.
\end{abstract}

Palavras-chave: Alimento vivo. Dieta artificial. Larvicultura.

\begin{abstract}
The objective of this work was to evaluate the performance of Rhinelepis aspera post larvae submitted to two diets. Completely randomized design with two treatments and eight repetitions were used. The stock density was seven post larvae per liter. The diets were composed by A, powder ration with $55 \%$ $\mathrm{CP}$ offered in organza sachets and B, 850 Artemia nauplii supplied to each post larvae three times a day. There was no significant difference between treatments in survival $(P \geq 0,05)$, but there was in biometry. The ration offered in sachets induced the highest averages in length $(\mathrm{mm})$ and weight $(\mathrm{mg})$, whilst the "natural diets" induced higher values of the condition factor. The results show that the ration sachets can be a promising alternative to larvae culture of $R$. aspera.
\end{abstract}

Key words: Alive food. Artificial diet. Larviculture.

1 Pós-Graduando do Curso de Mestrado em Zootecnia, Área de Concentração Produção Animal, Universidade Federal do Rio Grande do Sul, UFRGS. E-mail: luisbandeirantes@hotmail.com

2 Biólogo Graduado na Universidade Estadual do Norte do Paraná, Campus Cornélio Procópio, UENP. E-mail: jefersondiasdias2008@hotmail

3 Pós-Graduando do Curso de Doutorado em Zootecnia, Universidade Estadual de Maringá, UEM. E-mail: drfornari@hotmail. com

4 Prof. Associado do Departamento de Zootecnia da Universidade Estadual de Maringá, UEM. E-mail: rpribeiro@uem.br

5 Prof. Assistente do Departamento de Ciências Biológicas da Universidade Estadual do Norte do Paraná, UENP,Campus Luiz Meneghel. E-mail: marcoantoniobio@msn.com

* Autor para correspondência 


\section{Introdução}

O cascudo preto, Rhinelepis aspera, pertence à ordem Siluriformes e à família Loricariidae, possui hábito alimentar iliófago, atua na biocenose bentônica, operando na fase de pré mineralização da matéria orgânica presente no perifíton, acelerando a reciclagem de nutrientes (ANTONIUTTI et al., 1985). De acordo com Agostinho, Barbieri e Verani (1991), R. aspera é altamente suscetível às ações antrópicas como sobrepesca, poluição e construção de barragens. A combinação destes fatores tem ocasionado a diminuição nos estoques naturais da população, colocando o cascudo preto na lista do livro vermelho da fauna ameaçada no estado do Paraná, na categoria de vulnerável, e na lista das espécies presumivelmente ameaçadas de extinção no estado de Minas Gerais (LINS et al., 1997).

Antoniutti et al. (1985), destacaram que $R$. aspera tem grande potencial para ser explorado comercialmente, tanto como peixe ornamental, como também na produção, sendo fonte de proteína animal para alimentação humana, possuindo carne de textura firme não apresentando espinhos intramusculares, e de fácil adaptação 'as dietas artificiais. Além disso, o cascudo preto se caracteriza por ser um peixe rústico, de fácil adaptação a ambientes lênticos. Entretanto, a sua larvicultura ainda é o principal problema a ser solucionado, principalmente no que diz respeito à primeira alimentação.

Soares et al. (1997), afirmaram que muitos problemas relacionados ao cultivo de peixes já foram solucionados, porém, a etapa da larvicultura ainda apresenta muitas dificuldades e insucessos. Um dos principais problemas associado a esta fase é a alimentação das pós larvas. Kubitza (2004) relatou que a maioria das pós larvas das espécies nativas possuem trato digestório rudimentar ou incompleto, ou seja, apresentam ausência de algumas enzimas digestivas e, desta forma não conseguem utilizar alimentos artificiais de imediato. Estas pós larvas necessitam ingerir organismos vivos como primeiro alimento, pois as enzimas presentes nestes organismos auxiliam a digestão do alimento ingerido e estimulam o desenvolvimento do trato digestório das pós larvas.

Segundo Guevara (2003), as pós larvas podem ser divididas em precociais e altriciais, as pós larvas altriciais apresentam trato digestório indiferenciado, utilizando as enzimas das presas, já as precociais apresentam trato digestório diferenciado, portanto, são menos dependentes de alimentos vivos para o processo de digestão. $\mathrm{O}$ autor supracitado sugeriu que estudos morfológicos da ontogenia do cascudo preto devem ser conduzidos, pois, as pós larvas da espécie ainda não foram classificadas como altriciais ou precociais

O objetivo deste trabalho foi avaliar o desempenho de pós larvas de $R$. aspera submetidas a dois tipos de dietas: naúplios de artemia e ração em pó com $55 \%$ de proteína bruta fornecida em saches de organza.

\section{Material e Métodos}

O experimento foi conduzido na Estação de Hidrobiologia e Aqüicultura da Duke Energy do Brasil (coordenadas 22 53', 34" S e $49^{\circ} 59^{\prime}$ e $09^{\circ} \mathrm{O}$ ), localizada no município de Salto grande - SP, entre o período de 16 de fevereiro a 02 de março de 2009. Foram utilizadas 3584 pós larvas de cascudo preto, obtidas de matrizes mantidas em cativeiro na própria estação que foram induzidas à reprodução utilizando extrato bruto de hipófises de carpa comum (Cyprinus carpio) (EBHC), segundo a técnica proposta por Woynarovich e Horváth (1983).

Após a fertilização, os ovos foram transferidos para incubadoras do tipo "Israelense" de 7,5 litros, sendo que a eclosão dos ovos ocorreu após 36 horas. Foi realizado o monitoramento do desenvolvimento larval com o auxílio de um microscópio estereoscópico (aumento 20 de vezes), para determinar o momento da abertura da boca, quando 
uma amostra de 36 pós larvas foi fixada em formol $4 \%$, tamponado para posterior biometria. As demais pós larvas foram transferidas para as unidades experimentais, constituídas de incubadoras do tipo "calha" com volume total aproximado de 200 litros cada, subdividida em três compartimentos de 32 litros e com as laterais e fundo revestidos por tela.

O delineamento experimental foi o inteiramente casualizado com dois tratamentos e oito repetições. A densidade de estocagem foi de sete pós larvas/ litro. Foram utilizados dois tipos de dietas: A, ração em pó com $55 \%$ de proteína bruta oferecida em saches de tecido do tipo organza, sendo um por repetição, com aproximadamente 20 centímetros de comprimento, cinco centímetros de diâmetro e 100 gramas de massa, os quais eram substituídos a cada três dias. Já no tratamento que empregou alimento vivo, dieta $\mathrm{B}$, foi oferecido 850 naúplios de artemia/pós larva, três vezes ao dia nos seguintes horários: 8:00, 13:30 e 18:30. Para a eclosão dos cistos de Artemia sp, estimativa dos naúplios e sua quantificação utilizou-se metodologia semelhante à descrita por López (2005).

As unidades experimentais foram mantidas com fluxo contínuo e vazão controlada de 0,5 litros/minuto. A água provinha do reservatório da hidroelétrica de Salto Grande - SP. Durante o experimento, o monitoramento da água das incubadoras foi realizado três vezes ao dia nos seguintes horários de 8:00, 13:30 e 18:30 (sempre antes das alimentações, nos casos dos tratamentos que utilizaram naúplios Artêmia), sendo efetuadas medidas de temperatura e oxigênio dissolvido por meio de um oxímetro da marca Digimed, e pH por meio de pHmetro da marca Biosystem modelo PH206. As medidas de amônia foram realizadas utilizando-se kit de análise de água da marca Alfakit. Após cada leitura das variáveis físico químicas foi efetuado a troca de $50 \%$ do volume da água de todas as incubadoras e o sifonamento dos resíduos acumulados no fundo.

Durante o experimento foram coletadas duas amostras de 12 pós larvas por repetição, sendo a primeira realizada 10 dias após o início do experimento e a segunda, 15 dias. As pós larvas foram anestesiadas com benzocaína $(0,01 / 100$ $\mathrm{mL}$ ) e fixadas em formol $4 \%$, tamponadas e armazenadas em tubos de ensaio de $30 \mathrm{~mL}$ e acondicionadas em geladeira a uma temperatura $5^{\circ} \mathrm{C}$ para posterior biometria, antes do aferimento das medidas as pós larvas foram colocadas em papel de filtro para absorção do excesso de solução fixadora. Posteriormente, foram tomadas medidas de comprimento total e comprimento padrão com o auxílio de um paquímetro digital, e peso úmido por meios de balança analítica digital.

Ao final do experimento foram analisadas a sobrevivência e o peso médio, segundo Takata (2007), e o fator de condição, segundo Vazzoler (1996) por meio da expressão: $\mathrm{K}=\mathrm{Peso} /$ Comprimento $^{\mathrm{b}}$.

$\mathrm{O}$ fator de condição das pós larvas $(\mathrm{K})$, foi obtido por intermédio da curva potencial ajustada aos dados de peso e comprimento de cada tratamento pela seguinte expressão:

$y=a x^{b}$. O valor de b foi obtido pela logaritimização dos dados de peso e de comprimento total de todas as larvas amostradas em todas as biometrias. Esses dados foram plotados em gráfico e o valor de $b$, foi obtido através da equação linear: $\mathrm{y}=\mathrm{a}+\mathrm{bx}$

Utilizaram-se todos os dados das biometrias para se obter um único valor de $b$, representativo para cada tipo de dieta (artificial e natural) de todas as fases de desenvolvimento dos animais. Com esse procedimento os valores de fator de condição ficaram sujeitos apenas à variação do peso e do comprimento total dos animais.

Aos resultados foi aplicado o teste $\mathrm{F}$ de médias ANOVA, utilizando-se o software "Sistema para Análise e Separação de médias em experimentos Agrícolas" Versão 8.2 (CANTERI et al., 2001). Para comparação das médias foi utilizado o teste de Scott - Knott, o qual foi empregado a significância 
de $5 \%$.

\section{Resultados e Discussão}

As variáveis físico químicas observadas nesse experimento estão descritas na (Tabela 1), e estão dentro dos padrões recomendados para espécies tropicais estando de acordo com o descrito por Baldisserotto (2002).

Tabela 1. Valores médios de temperatura, $\mathrm{pH}$, oxigênio dissolvido (OD) e amônia.

\begin{tabular}{lcc}
\hline Parâmetros físico-químicos & Ração comercial & Naúplios de Artemia \\
\hline Temperatura $\left({ }^{\circ} \mathrm{C}\right)$ & $27,11 \pm 1,09$ & $27,09 \pm 1,10$ \\
$\mathrm{pH}$ & $6,38 \pm 0,50$ & $6,51 \pm 0,54$ \\
$\mathrm{OD}\left(\mathrm{mg} \mathrm{L}^{-1}\right)$ & $5,74 \pm 0,79$ & $6,19 \pm 0,59$ \\
Amônia & $0,32 \pm 0,08$ & $0,26 \pm 0,10$ \\
\hline
\end{tabular}

Desvio Padrão ( \pm ).

Após 15 dias de cultivo, a análise dos resultados de sobrevivência das pós larvas de cascudo preto não apresentou diferenças significativas $(\mathrm{P} \geq 0,05)$ entre os tratamentos. A (Figura 1) apresenta os resultados de sobrevivência das pós larvas do tratamento que utilizou ração oferecida em saches comparado com o tratamento que utilizou naúplios de artemia.

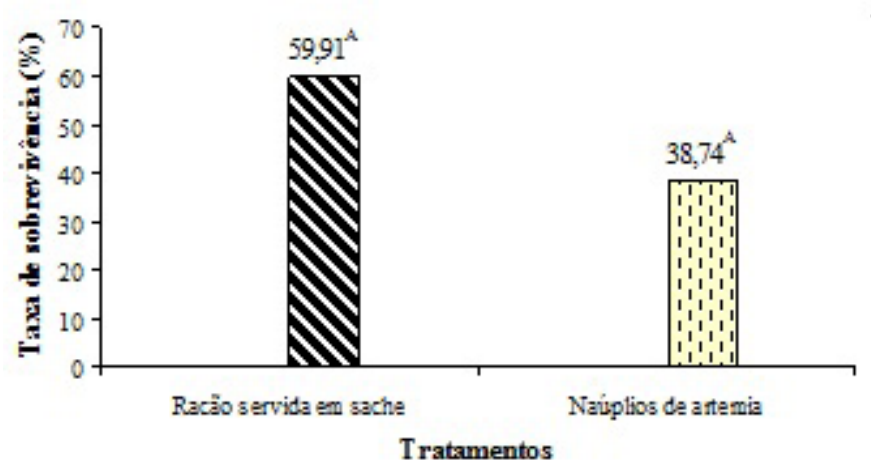

Figura 1. Valores percentuais médios de sobrevivência do Rhinelepis aspersa nos tratamentos que utilizaram ração em saches e naúplios de Artemia $(\mathrm{CV}=24,57 \%)$

Vários estudos foram realizados com o objetivo de avaliar a utilização de dietas artificiais (ração seca) e alimentos vivos (naúplios de artemia, zooplâncton e outros) na larvicultura, para o quinguio Carassius auratus (SOARES; HAYASHI; GONÇALVES, 2000), o peixe rei Odontesthes bonariensis (PIEDRAS; POUEY, 2004), o mandi amarelo Pimelodus maculatus (LUZ; ZANIBONI FILHO, 2001) e a piracanjuba Brycon orbignyanus (PIOVEZAN, 1994) e em todos os experimentos supracitados, os tratamentos que utilizaram alimentos vivos apresentaram resultados de sobrevivência superiores aos tratamentos que utilizaram dietas artificiais fornecidas diretamente 'as pós larvas. Estes resultados são contrastantes aos obtidos no presente estudo, indicando que as pós larvas de Rhinelepis aspera obtiveram aproveitamento estatisticamente semelhante com as fontes alimentares (ração e naúplios de artemia).

A análise dos resultados da primeira biometria 
revelou existir diferenças significativas $(\mathrm{P} \geq 0,05)$, entre as duas dietas, sendo que as pós larvas do tratamento que empregou ração oferecida em saches obteve as maiores médias de comprimento $(\mathrm{mm})$ e peso (mg). O mesmo foi observado na segunda biometria onde a dieta artificial proporcionou às pos larvas, médias significativamente maiores $(\mathrm{P} \geq 0,05)$ para peso e comprimento. A (Tabela 2) apresenta os valores médios de peso e comprimento.

A (Figura 2) representa a relação entre peso e comprimento das pós larvas de Rhinelepis aspera ao final do experimento.

Tabela 2. Valores médios de comprimento ( $\mathrm{mm})$, peso (mg) e fator de condição das pós larvas de Rhinelepis aspera alimentadas com ração oferecida em saches e naúplios de Artemia.

\begin{tabular}{lccc}
\hline Variáveis & Ração comercial & Naúplios de Artemia & CV (\%) \\
\hline Comprimento médio $(\mathrm{mm}) 1^{\circ}$ Biometria & $15,62 \mathrm{~A} \pm 1,02$ & $13,95 \mathrm{~B} \pm 0,98$ & 7,05 \\
Peso médio $(\mathrm{mg}) 1^{\circ}$ Biometria & $60,05 \mathrm{~A} \pm 1,43$ & $43,87 \mathrm{~B} \pm 1,26$ & 18,36 \\
Comprimento médio $(\mathrm{mm}) 2^{\circ}$ Biometria & $22,13 \mathrm{~A} \pm 1,12$ & $16,63 \mathrm{~B} \pm 1,34$ & 8,30 \\
Peso médio (mg) $2^{\circ}$ Biometria & $163,61 \mathrm{~A} \pm 5,98$ & $53,35 \mathrm{~B} \pm 3,87$ & 24,00 \\
Fator de condição & $0,0201 \mathrm{~B} \pm 0,001$ & $0,0371 \mathrm{~A} \pm 0,0067$ & 16,95 \\
\hline
\end{tabular}

Médias seguidas de letras diferentes na linha diferem entre si $(\mathrm{P}<0,05)$. Desvio padrão $( \pm)$.

Os valores médios de peso e comprimento das pós larvas do tratamento que empregou ração em saches são contraditórios aos resultados obtidos por Luz e Zaniboni Filho (2001), em mandi amarelo (Pimelodus maculatus); Piedras e Pouey (2004) em experimento com o peixe rei (Odontesthes bonariensis); Feiden, Hayashi e Boscolo (2006) em estudo com o surubim do Iguaçu (Steindachneridion melanodermatum), onde a utilização de alimento vivo apresentou melhor desempenho comparado a dieta artificial. A possível causa para esta contradição pode ser explicada pelo fato do $R$. aspera possuir hábito alimentar iliófago ou detritívoro e natação menos ativa (ANTONIUTTI et al., 1985), quando comparado `as espécies citadas, portanto, a captura de alimento vivo na coluna d'água seria um procedimento com maior custo energético em relação ao comportamento observado no processo de alimentação das pós larvas nos saches. Esta hipótese pode ser confirmada por meio de estudos de conteúdo estomacal do $R$. aspera realizados por Delariva e Agostinho (2001), mostrando que a espécie possui dieta constituída de detritos orgânicos, organismos perifíticos e sedimento.

Apesar do desconhecimento das exigências nutricionais específicas de $R$. aspera, em estudos de crescimento realizados por López (2005), onde foram empregados diferentes taxas de oferta de alimento, observou-se que houve uma boa aceitação da dieta artificial pelo cascudo preto, com resultados de crescimento satisfatórios para o cultivo.

Durante o cultivo observou se que à medida que as pós larvas aumentaram o comprimento, houve maior variabilidade de peso, no tratamento que empregou ração oferecida em saches. Os resultados da última biometria nos tratamentos que utilizaram dieta artificial apresentaram considerável acréscimo de peso e comprimento. Estes resultados foram semelhantes aos obtidos por López (2005) em pós larvas da mesma espécie. 

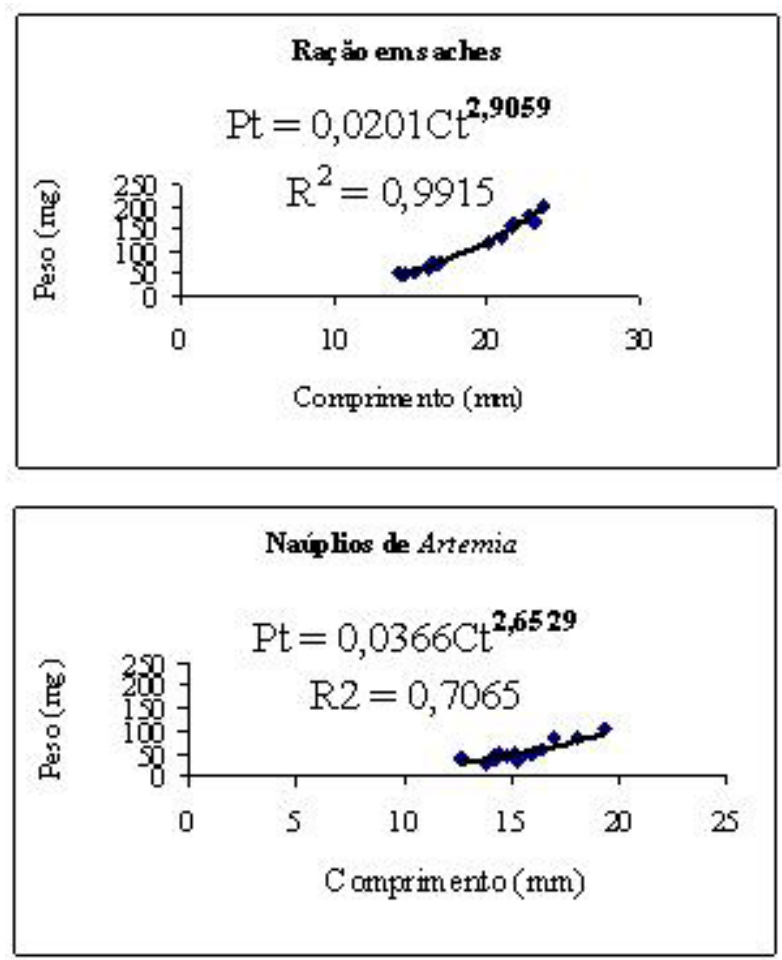

Figura 2. Relação entre peso e comprimento das pós larvas de Rhinelepis aspera ao final do experimento.

Para o cálculo do fator de condição das pós larvas adotou se valores médios de b (coeficiente de regressão) diferentes, pois as pós larvas foram submetidas a diferentes condições de alimentação. As pós larvas do tratamento que empregou dieta artificial alcançaram valor médio de $b=2,9059$, portanto, ligeiramente inferior a 3,0, o que indica um crescimento isométrico. Já, o tratamento em que se utilizou naúplios de artemia, o valor médio de $b$ obtido foi inferior a 3,0 $(b=2,6529)$ sugerindo um crescimento alométrico.

De acordo com Rocha et al. (2005), a relação entre o peso e o comprimento corporal pode apresentar condições de crescimento isométrico quando $\mathrm{b}=3,0$; isto é, o peso aumenta com o comprimento elevado ao cubo. Por outro lado, quando $b<3,0$ o incremento é devido ao peso, e quando $b>3,0$ o incremento é devido ao comprimento. O fator de condição permite comparações entre populações de peixes que estão submetidas a diferentes condições de clima, temperatura, alimentação e densidade do peixe.

A comparação dos resultados médios do fator de condição mostrou que a "dieta natural" alcançou valores superiores $(\mathrm{P} \geq 0,05)$ em relação à "dieta artificial", sugerindo melhores condições de "bem estar" nos tratamentos que utilizaram naúplios de artemia. A possível causa para os resultados observados pode estar relacionada ao fato do tratamento que utilizou como dieta, alimento vivo, ter obtido menores densidades de estocagem nas repetições quando comparado ao tratamento que utilizou dieta artificial, aumentando a disponibilidade de alimento. Este resultado é semelhante ao encontrado por Zuanon, Assan e Fernandes (2004), avaliando o desempenho de Trichogaster trichopterus em diferentes densidades de estocagem, onde os resultados superiores de fator de condição foram observados nas menores densidades. Os valores médios de fator de condição 
das pós larvas de cascudo preto estão apresentados na Tabela 02.

Sousa (2005), trabalhando com pintado (Pseudoplatystoma corruscans) em diferentes sistemas de produção, obteve as melhores condições de "bem estar" no sistema de produção semi-intensivo onde as densidades de estocagem são inferiores às praticadas pelo sistema intensivo de produção.

\section{Conclusão}

A utilização de saches para o fornecimento de ração pode ser uma boa alternativa de manejo na larvicultura do Rhinelepis aspera, mas estudos complementares devem ser realizados para melhorar a sua utilização. Desta forma, sugere se o desenvolvimento de experimentos que avaliem a quantidade de ração fornecida nos saches, assim como as dimensões e formato dos saches e seus efeitos sobre a sobrevivência, ganho de peso e fator de condição das pós larvas.

\section{Agradecimentos}

À Duke energy do Brasil por ceder toda estrutura física de sua estação de hidrobiologia e aqüicultura e material biológico sem os quais este estudo não poderia ser realizado.

\section{Referências}

AGOSTINHO, A. A.; BARBIERI, G.; VERANI, J. $\mathrm{R}$. Idade de crescimento do cascudo preto Rhinelepis aspera (SILURIFORMES, LORICARIIDAE) no Rio Paranapanema, Bacia do Rio Paraná. Revista UNIMAR, Maringá, v. 2, n. 13, p. 49-258, out. 1991.

ANTONIUTTI, D. M.; RANZANI-PAIVA, T. M. J. ; GODINHO, H. M.; PAIVA, P. Relação peso total/ comprimento, crescimento do cascudo Plecostomus albopunatatos Regan, 1908 (Osteichthyes, Loricariidae) do rio Jaquari. Boletim do Instituto de Pesca, São Paulo, SP, v. 12, n. 3, p. 105-120, 1985.

BALDISSEROTTO, B. Fisiologia de peixes aplicada a piscicultura. UFSM, Santa Maria, RS, 2002. 212 p.

CANTERI, M. G.; ALTHAUS, R. A.; VIRGENS FILHO, J. S.; GIGLIOTI, E. A.; GODOY, C. V. SASM - Agri: Sistema para análise e separação de médias em experimentos agrícolas pelos métodos Scoft - Knott, Tukey e Duncan. Revista Brasileira de Agrocomputação, Ponta Grossa, v. 1, n. 2, p. 18-24, 2001.

DELARIVA, R. L.; AGOSTINHO, A. A. Relationship between morphology and diets of six neotropical loriicads. Journal Fish Biology. London, v. 58, n. 3, p. 832-847, 2001.

FEIDEN, A.; HAYASHI, C.; BOSCOLO, W. Desenvolvimento de larvas de surubim-do-iguaçu (Steindachneridion melanodermatum) submetidas a diferentes dietas. Revista. Brasileira de Zootecnia, Viçosa, MG, v. 35, n. 6, p. 2203-2210, 2006.

GUEVARA, M. J. P. Enriquecimento de zooplâncton com óleo de peixe na larvicultura do pacu, Piaractus mesopotamicus curimbatá Prochilodus lineatus. 2003. Dissertação (Mestrado em Zootecnia) - Universidade Federal de Lavras, Lavras.

KUBITZA, F. Reprodução, larvicultura e produção de alevinos de peixes nativos. Jundiaí, SP: Aqua Supre Com.Suprim. Aquicultura Ltda, 2004. 38 p.

LINS, L. V.; MACHADO, A. B. M.; COST, C. M. R.; HERRMANN, G. Roteiro metodológico para elaboração de listas de espécies ameaçadas de extinção (contendo a lista oficial de fauna ameaçada de extinção de Minas Gerais). Belo Horizonte, MG: Fundação Biodiversitas, 1997. 7 p. (Publicações Avulsas da Fundação Biodiversitas).

LÓPEZ, C. M. Crescimento de larvas de cascudo preto (Rhinelepis áspera) Spix \& Agassiz, 1829 (Osteichthyies: Siluriforme, Loricariidae) submetidas a diferentes níveis alimentares. 2005. Dissertação (Mestrado em Aqüicultura) - Universidade Estadual Paulista. Centro de Aqüicultura, Jaboticabal, São Paulo.

LUZ, R. K.; ZANIBONI FILHO, E. Utilização de diferentes dietas na primeira alimentação do mandi amarelo (Pimelodus maculatus, Lacépéde). Acta Scientiarum. Animal Sciences, Maringá, v. 23, n. 2, p. 483-489, 2001.

PIEDRAS, S. R. N.; POUEY, J. L. O. F. Alimentação de alevinos de peixe-rei (Odontesthes bonariensis) com dietas naturais e artificiais. Ciência Rural, Sana Maria, RS, v. 34, n. 4, p. 1203-1206, 2004.

PIOVEZAN, U. Efeito da dieta na sobrevivência de larvas de piracanjuba (Brycon orbignyanus) - CAUNESP. In: SEMINÁRIO SOBRE CRIAÇÃO DE ESPÉCIES 
DO GÊNERO BRYCON, 1., 1994, Pirassununga, SP. Anais... Pirassununga, SP: CEPTA/IBAMA, 1994. p. 1718.

ROCHA, M. A.; RIBEIRO, E. L. de A.; MIZUBUTI, I. Y.; SILVA, L. das D. F. da; BOROSKY, J. C.; RUBIN, K. C. P. Uso do fator de condição alométrico e de fulton na comparação de carpa (Cyprinus carpio), considerando os sexos e idade. Semina: Ciências Agrárias, Londrina, PR, v. 26, n. 3, p. 429-434, jul./set. 2005.

SOARES, C. M.; HAYASHI, C.; GONÇALVES, G. S. Plâncton, Artemia sp, dieta artificial e suas combinações no desenvolvimento e sobrevivência do quinguio (Carassius auratus) durante a larvicultura. Acta Scientiarum. Animal Sciences, Maringá, v. 22, n. 2, p. 383-388, 2000.

SOARES, C. M.; HAYASHI, C.; FURUYA, W. M.; FURUYA, V. R. B.; MARANHÃO, T. C. F. Alimentação natural de larvas do cascudo preto (Rhinelepis aspera, V.) em tanques de cultivo. Boletim do Instituto de Pesca, São Paulo, v. 24, p. 109-117, 1997. (Especial).

SOUSA, A. D. L. Efeito dos sistemas de criação semi-intensivo (viveiro escavado) e intensivo (tanquerede) no desenvolvimento produtivo do pintado, Pseudoplatystoma corruscans (spix \& agassiz, 1829) (Siluriformes: pimelodidae). 2005. Dissertação (Mestrado em Aqüicultura) - Centro de Aqüicultura da UNESP.Campus de Jaboticabal, São Paulo.

TAKATA, R. Produção de juvenis de artemia franciscana $e$ análise da utilização de dietas vivas e inertes na larvicultura intensiva do pintado Pseudoplatystoma coruscans. 2007. Dissertação (Mestrado em Aqüicultura) - Centro de Aqüicultura da Universidade Estadual Paulista. Centro de Aqüicultura, Jaboticabal, São Paulo.

VAZZOLER, A.E. A. M. Biologia da reprodução de peixes teleósteos: teoria e prática. Maringá: Eduem, 1996.

WOYNAROVICH, E.; HORVÁTH, L. A propagação artificial de peixes de águas tropicais: manual de extenção. CHAMA, V. L. M. (Trad.). Brasília: FAO/ CODEVASF/CNPQ, 1983. p. 225.

ZUANON, J. A. S.; ASSAN, M.; FERNANDES, J. B. K. Desempenho de Tricogaster (Trichogaster trichopterus) submetido a diferentes níveis de arraçoamento e densidades de estocagem. Revista Brasileira de Zootecnia, Viçosa, MG, v. 33, n. 6, p. 1639-1645, 2004. 